\title{
The VIRMOS deep imaging survey
}

\section{Overview, survey strategy, and CFH12K observations ${ }^{\star}$}

\author{
O. Le Fèvre ${ }^{1}$, Y. Mellier ${ }^{2,3}$, H. J. McCracken ${ }^{1,4}$, S. Foucaud ${ }^{1}$, S. Gwyn ${ }^{1}$, M. Radovich ${ }^{2,5}$, M. Dantel-Fort ${ }^{3}$, \\ E. Bertin ${ }^{2,3}$, C. Moreau ${ }^{1}$, J.-C. Cuillandre ${ }^{6}$, M. Pierre ${ }^{7}$, V. Le Brun ${ }^{1}$, A. Mazure ${ }^{1}$, and L. Tresse ${ }^{1, \star \star}$ \\ ${ }^{1}$ Laboratoire d'Astrophysique de Marseille, UMR 6110, CNRS-Université de Provence, Traverse du Siphon-Les trois Lucs, \\ 13012 Marseille, France \\ 2 Institut d'Astrophysique de Paris, UMR 7095, 98bis Bvd Arago, 75014 Paris, France \\ 3 Observatoire de Paris, LERMA, UMR 8112, 61 Av. de l'Observatoire, 75014 Paris, France \\ 4 Osservatorio Astronomico di Bologna, via Ranzani 1, 40127 Bologna, Italy \\ 5 Osservatorio di Capodimonte, via Moiariello 16, 80131 Napoli, Italy \\ ${ }^{6}$ Canada-France Telescope Corporation, 65-1238 Mamalahoa Hwy, Kamuela, Hawaii 96743, USA \\ 7 Service d'Astrophysique, CE Saclay, L'Orme des Meurisiers, 91191 Gif-sur-Yvette Cedex, France
}

Received 12 June 2003 / Accepted 19 December 2003

\begin{abstract}
This paper describes the CFH12K-VIRMOS survey: a deep BVRI imaging survey in four fields totalling more than $17 \mathrm{deg}^{2}$, conducted with the $40 \times 30 \operatorname{arcmin}^{2}$ field CFH-12K camera. The survey is intended to be a multi-purpose survey used for a variety of science goals, including surveys of very high redshift galaxies and weak lensing studies.

Four high galactic latitude fields, each $2 \times 2 \mathrm{deg}^{2}$, have been selected along the celestial equator: 0226-04, 1003+01, 1400+05, and $2217+00$. The $16 \mathrm{deg}^{2}$ of the "wide" survey are covered with exposure times of $2 \mathrm{hr}, 1.5 \mathrm{hr}, 1 \mathrm{hr}, 1 \mathrm{hr}$, respectively while the $1.3 \times 1 \mathrm{deg}^{2}$ area of the "deep" survey at the center of the 0226-04 field is covered with exposure times of $7 \mathrm{~h}, 4.5 \mathrm{~h}, 3 \mathrm{~h}$, $3 \mathrm{~h}$, in $B V R I$ respectively. An additional area $\sim 2 \mathrm{deg}^{2}$ has been imaged in the 0226-04 field corresponding to the area surveyed by the XMM-LSS program (Pierre et al. 2003).

The data is pipeline processed at the Terapix facility at the Institut d'Astrophysique de Paris to produce large mosaic images. The catalogs produced contain the positions, shapes, total and aperture magnitudes for 2.175 million objects measured in the four areas. The limiting magnitudes, measured as a $5 \sigma$ measurement in a $3 \operatorname{arcsec}$ diameter aperture is $I_{\mathrm{AB}}=24.8$ in the "Wide" areas, and $I_{\mathrm{AB}}=25.3$ in the deep area. Careful quality control has been applied on the data to ensure internal consistency and assess the photometric and astrometric accuracy as described in a joint paper (McCracken et al. 2003). These catalogs are used to select targets for the VIRMOS-VLT Deep Survey, a large spectroscopic survey of the distant universe (Le Fèvre et al. 2003). First results from the CFH12K-VIRMOS survey have been published on weak lensing (e.g. van Waerbeke \& Mellier 2003). Catalogs and images are available through the VIRMOS database environment under Oracle (http://www . oamp. fr/cencos). They are open for general use since July 1st, 2003.
\end{abstract}

Key words. cosmology: observations - galaxies: evolution - cosmology: gravitational lensing - cosmology: large-scale structure of universe

\section{Introduction}

Deep imaging over large areas is required for many fields of astronomy to survey large numbers of objects or to search for rare objects. It is a key tool to furthering our understanding

Send offprint requests to: $\mathrm{O}$. Le Fèvre, e-mail: olivier.lefevre@oamp. fr

* Appendix A is only available in electronic form at http://www . edpsciences.org

$\star \star$ The data presented in this paper has been obtained with the Canada-France-Hawaii Telescope, operated by the National Research Council of Canada, the Centre National de la Recherche Scientifique of France, and the University of Hawaii. of the Universe, from solar system studies to the most distant galaxies. The measurement of positions, magnitudes, colors, and shape are key observables to all astronomical investigations. From astrometric and photometric measurements, a census of the number and positions of various classes of objects can be conducted as a function of magnitude, color, or shape.

Progress in detector area have allowed the design and construction of large area CCD mosaics covering a significant fraction of the available field of view on large telescopes (see Groom 2000, for a review). These mosaics have enabled survey work on large areas which had only been possible previously using photographic detectors. Probing scales larger than 0.5 degree in one single exposure with the sensitivity of CCDs 
Table 1. Survey fields.

\begin{tabular}{llllllc}
\hline \hline Field & $\begin{array}{l}\alpha_{2000} \\
\text { center }\end{array}$ & $\begin{array}{l}\delta_{2000} \\
\text { center }\end{array}$ & $b$ & $l$ & $E(B-V)$ & $\begin{array}{c}\text { fieldsize } \\
\text { sq. deg. (I-band) }\end{array}$ \\
\hline $0226-04$ “wide" (\& XMM - LSS) & $02^{\mathrm{h}} 24^{\mathrm{m}} 39.75^{\mathrm{s}}$ & $-04^{\circ} 30^{\prime} 00^{\prime \prime}$ & -58.0 & 172.0 & 0.027 & 5.4 \\
$0226-04$ “deep" & $02^{\mathrm{h}} 26^{\mathrm{m}} 00^{\mathrm{s}}$ & $-04^{\circ} 30^{\prime} 00^{\prime \prime}$ & & & & 1.45 \\
$1003+01$ & $10^{\mathrm{h}} 03^{\mathrm{m}} 39 . .00^{\mathrm{s}}$ & $+01^{\circ} 54^{\prime} 39^{\prime \prime}$ & 42.6 & 237.8 & 0.023 & 4.14 \\
$1400+05$ & $14^{\mathrm{h}} 00^{\mathrm{m}} 00.00^{\mathrm{s}}$ & $+05^{\circ} 00^{\prime} 00^{\prime \prime}$ & 62.5 & 342.4 & 0.026 & 4.32 \\
$2217+00$ & $22^{\mathrm{h}} 17^{\mathrm{m}} 50.40^{\mathrm{s}}$ & $+00^{\circ} 24^{\prime} 27^{\prime \prime}$ & -44.0 & 63.3 & 0.062 & 3.60 \\
\hline
\end{tabular}

has become possible at a few facilities in the past few years (Cuillandre et al. 2000; Boulade et al. 2000; Miyazaki et al. 2002; Kuijken et al. 2002), enabling a wealth of new deep survey initiatives (Postman et al. 1998; Nonino et al. 1999; McCracken et al. 2001; McMahon et al. 2001; Wilson 2003)

This paper is the first of a series that describes the deep survey we started when the CFH12K camera became available at CFHT in 1999. The main survey covers a total of $16 \mathrm{deg}^{2}$ in four areas, each $2 \times 2 \mathrm{deg}^{2}$, which have been imaged in $B, V, R$ and $I$ bands, at a depth equivalent to $I_{\mathrm{AB}}=24.8$ for a $3 \sigma$ detection in a 3 arcsec diameter circular aperture for all the area, with a deeper area imaged to $I_{\mathrm{AB}}=25.3$. In this paper, we focus on the survey goals and strategy, and the observations performed, together with an overview of the pipeline processing and the content of the photometric catalogs. Other papers in this series (McCracken et al. 2003; Gwyn et al. 2004, in preparation) describe in detail the procedures followed to construct the astrometry and photometry of the large image mosaics, and the quality control applied. The VIRMOS survey has been used already for cosmic shear studies and eight refereed papers have been published so far from this data set by the VIRMOS-DESCART project (see Waerbeke \& Mellier 2003, for a review).

\section{Survey goals}

The survey has been designed to address a broad range of astrophysical questions in one single observing strategy. Several main science drivers have been identified:

- study the evolution of galaxies from redshifts $\sim 5$;

- study the evolution of large scale structures over $100 h^{-1}$ Mpc from redshifts $\sim 5$;

- measure weak lensing signature of large scale structures;

- measure the properties of galaxy biasing using together the dark matter (from weak lensing) and the galaxy distribution;

- identify new high redshift clusters of galaxies;

- identify faint AGN and study their evolution;

- identify new Kuiper belt objects to give new insights into the formation of the solar system;

- identify very faint halo white dwarfs;

- study high redshift Lyman-break galaxies with $3.5<z<$ 4.2; using the multi-color data set of the optical and $U$-band survey.

This data has also been time-sequenced to search for high redshift supernovae.
This imaging survey is being used to select the targets for the VLT-VIRMOS deep redshift survey of more than 100000 galaxies with $0<z<5+$ (Le Fèvre et al. 2003), to obtain large catalogues of galaxy shapes for cosmic shear studies and as the optical counterpart to the XMM medium deep survey (http://vela.astro.ulg.ac.be/themes/ spatial/xmm/LSS/) being carried out with XMM Pierre et al. (2003). The deep VIRMOS field is also complemented by a $U$-band follow up at ESO, with the WFI camera Radovich et al. (2003), as well as in radio wavelengths with the VLA Bondi et al. (2003). Around $150^{\prime 2}$ of the deep field has also been observed in $K$-band, with SOFI at the ESO/NTT Iovino et al. (2003).

\section{Survey strategy}

\subsection{Field selection}

Survey fields have been selected with the following criteria:

- along the celestial equator to allow for visibility from northern and southern hemisphere observatories;

- galactic latitude higher than $l=45 \mathrm{deg}$;

- low cirrus absorption as measured from the DIRBE maps available when the survey started in 1998;

- visibility of any two fields at any time of the year to fill observing nights.

The four fields selected are listed in Table 1 and their position in the dust sky map shown in Fig. 1.

\subsection{Survey depth}

The survey has been designed to reach a limiting magnitude $I_{\mathrm{AB}}=24.5$ (at $5 \sigma$ in a 3 arcsec diameter aperture) in all of the area surveyed, with a smaller $1.3 \mathrm{deg}^{2}$ area in the 0226-04 field observed to $I_{\mathrm{AB}}=25$. The depth of this imaging survey is then at least one magnitude deeper than the limiting magnitude(s) of the VIRMOS-VLT Deep Survey (VVDS), set to be $I_{\mathrm{AB}}=22.5$ for the wide areas and $I_{\mathrm{AB}}=24$ for the deep area (Le Fèvre et al. 2003), and ensures that the imaging survey will not introduce any bias in galaxy samples selected for the spectroscopic survey.

The actual measured completeness limits are presented in Sect. 6.1. 


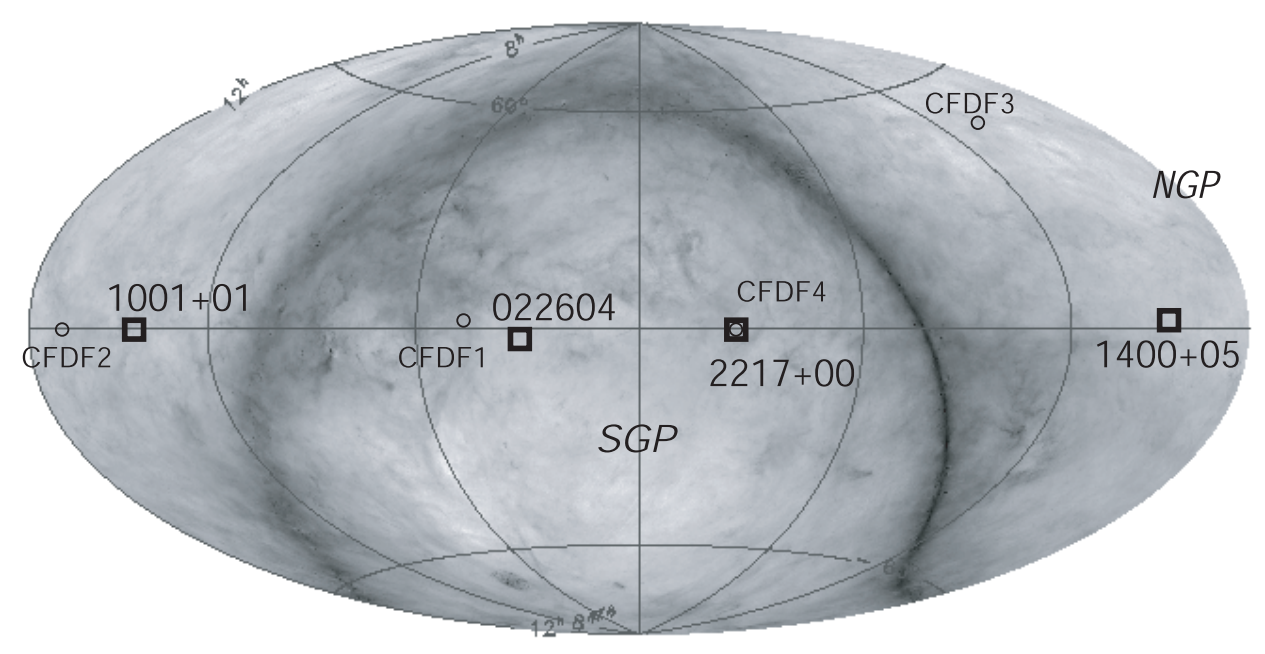

Fig. 1. Positions of the 4 VIRMOS fields on a dust map (Schlegel et al. 1998) of the sky (Aitoff projection in equatorial coordinates). The open squares are the center positions. One can see that the four fields are visible from CFHT as well as the ESO Paranal and the ESO La Silla observatories and are separated by at least $4 \mathrm{~h}$, making the observations optimally spread over the nights and over the year. The square size is not exactly scaled according to the true field size $\left(2^{\circ} \times 2^{\circ}\right)$ to ease visualization. For comparison the open circles indicate the center positions of the CFDF fields (McCracken et al. 2001). The CFDF field sizes are 16 times smaller than the VIRMOS. The dust map was generated using the Skyview virtual observatory tools (http://skys4.gsfc.nasa.gov/).

\subsection{The CFH12K camera}

Observations have been carried out with the CFHT-12K camera build by CFHT and the University of Hawaii (Cuillandre et al. 2000). The CFH12K camera (http://www . cfht.hawaii.edu/Instruments/Imaging/CFH12K/) is a particularly powerful system. It is based on a mosaic of 12 3-edge buttable MIT/Lincoln-Labs. CCID20 thinned backside illuminated CCDs, each $2048 \times 4096$ pixels for a total array size of $12288 \times 8192$ pixels $(18.4 \mathrm{~cm} \times 12.3 \mathrm{~cm}$ in physical size). The pixel size (15 microns) corresponds to a scale of $0.205 \mathrm{arcsec} / \mathrm{pixel}$ at CFHT prime focus, well adapted to the mean seeing at the CFHT prime focus $\left(\approx 0.8^{\prime \prime}\right)$. It covers a field of view $42 \times 28 \mathrm{arcmin}^{2}$. The camera is composed of two different types of CCDs. Three are high resisitivity chips and nine are epitaxial silicon devices. The high resistivity CCDs have better efficiency than the epitaxial beyond $500 \mathrm{~nm}$, but are less efficient in the blue (see Fig. 2). This difference is corrected during the pre-processing step that rescales each CCD with respect to a reference CCD (normally chip 7).

None of the CCDs has a readout noise higher than 6 electrons rms nor shows dark current signal over $1 \mathrm{~h}$ time scale, which is largely sufficient for deep imaging surveys with sky background noise dominated images. The readout time is about one minute, which permits the easy splitting of long exposures into short ones without increasing significantly the overheads. In addition, to facilitate the construction of master flat fields and fringe patterns and to simplify the cosmic ray rejection process, the image splitting also permits a better control of the camera focus and the rejection of bad seeing exposures. The autonomy of the LN2 reservoir is much longer than a full single night observation process and we never detected any temperature drift during the nights dedicated to the VIRMOS survey.
Standard Johnson-Kron-Cousins BVRI broad band filters are available on CFH12K. VRI are rather standard Mould filters, but $B$ is special (see Fig. 2), being somewhat redder than the standard $B$. They have been designed in order to cover the whole optical spectral range in a continuous manner. The photometric information have therefore no gaps from one band to the other allowing the probing of the whole redshift range from $z=0$ to $z=5$ continuously. This advantage is however obtained at the expense of photometric redshift accuracy for high$z$ galaxies.

\subsection{Observing strategy}

Each $2 \times 2$ deg $^{2}$ field has been divided in a series of CFH12K pointings covering the full area, with a slight overlap of $\sim 30$ arcsec between each pointing. A classical shift-and-add observing strategy was adopted, each pointing being observed between 5 and 12 times depending on the filters and depth. Because during our first two observing runs two of the twelve CCDs were of poor quality, the grid of pointings has had to be adjusted to the available pixels for these runs. We started operating with $35 \times 28 \operatorname{arcmin}^{2}$ fields, switching to the full $42 \times 28 \operatorname{arcmin}^{2}$ field when new CCDs were installed in the first observing period of 2000 . The field geometry is therefore non-rectangular in some bandpasses.

We aimed to bring first the $I$-band coverage to $2 \times 2 \mathrm{deg}^{2}$ in each of the fields to ensure that reference catalogs would be available for the magnitude selected VIRMOS-VLT Deep redshift Survey. We then completed the coverage in other bands in a uniform way, proportional to the actual number of clear nights. During the acquisition of a sequence of exposures, the aperture flux of control stars was monitored for changes in photometric conditions. The exposure times were lengthened in case flux loss was detected during sub-exposures. 


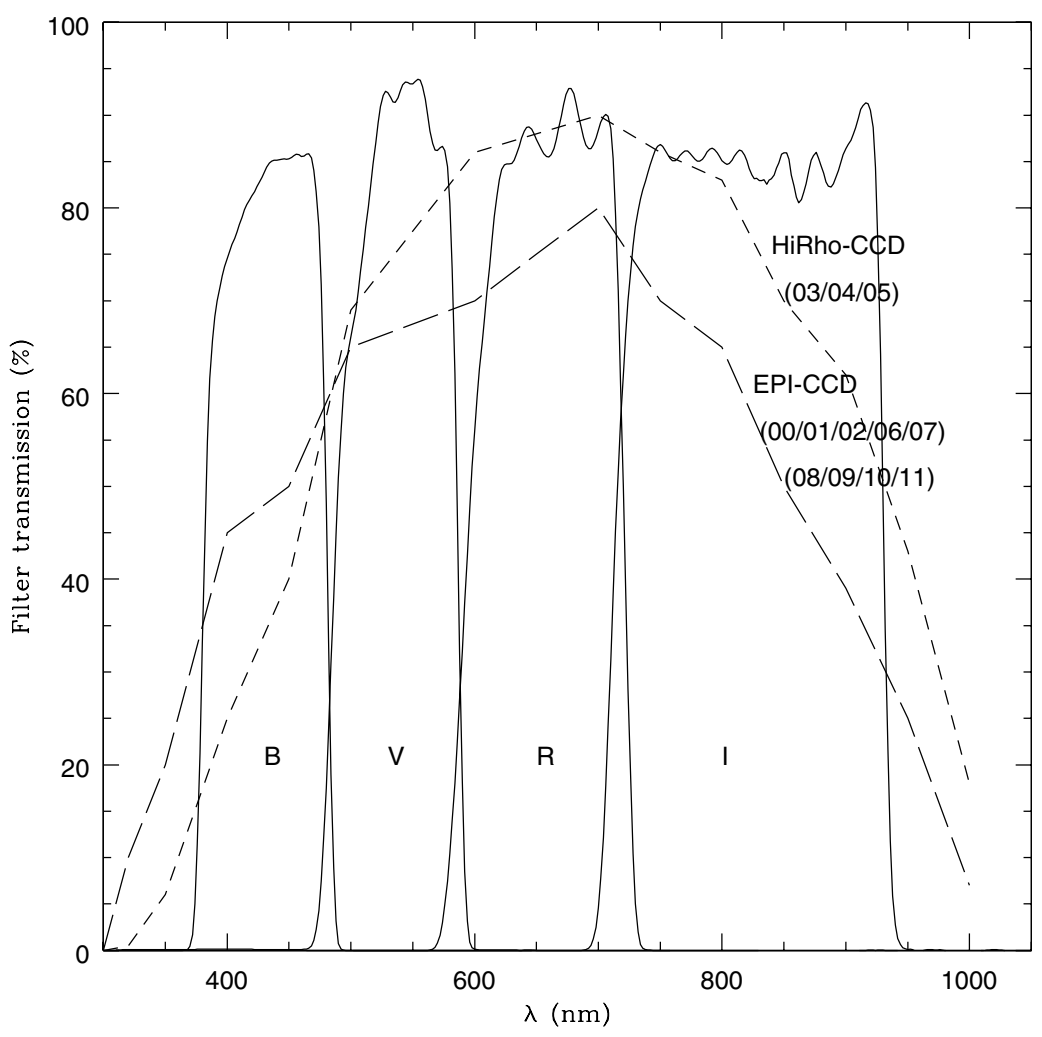

Fig. 2. Filter transmission and CCD quantum efficiency of the CFH12K camera used for the VIRMOS optical survey. The camera is composed of 9 epitaxial silicon (EPI) and three high restivity (HiRho) devices. Both devices have different quantum efficiency, as show by the long-dashed and short-dashed curves. The numbers indicate the CFHT reference number of the CFH12K CCDs.

\section{Observations}

\subsection{Observed pointings}

The individual pointings observed are listed in http://www.oamp.fr/virmos, with the date of observations, the $F W H M$ measured on point sources images on the detectors, and the corresponding CFHT archive files.

The final CFH12K mosaics resulting from the combination of these individual pointings are listed in Table 2 for each of the four fields and four bands, together with the resulting exposure times.

\subsection{Calibrations}

Standard photometric fields from Landolt (1992) were acquired each night survey observations were carried out. The fields SA92, SA101 and SA110 have been observed several times over the period 1999-2002 in order to check the reliability of the photometric calibration and the stability of the $\mathrm{CFH} 12 \mathrm{~K}$ performances. The detailed process of photometric calibration, relative from exposure to exposure within a set of exposures of a pointing in each bandpass, as well as the absolute flux calibration from the observed standards, is described in detail in (McCracken et al. 2003).

\subsection{Image quality}

The image quality of each exposure acquired for the survey is presented in Fig. 3 for each bands. The median seeing is
$F W H M \sim 0.75^{\prime \prime}$ in the $I$ band but increases to $0.88^{\prime \prime}$ in $V$ and $R$, and $0.97^{\prime \prime}$ in $B$. Moreover, the $V$ and $R$ seeing distributions are significantly broader than the $I$ and $B$ data sets. This trend reflects that atmospheric dispersion is negligible in $I$-band and increases towards the $B$-band. However, it mostly results from our observing strategy since we preferentially carried out $I$-band observations during good seeing periods in order to get high quality $I$-band selected catalogues for the VMOS spectroscopic sample as well as for the cosmic shear studies.

\section{Data processing}

\subsection{Pre-processing}

All data were pre-processed using the FLIPS software package developed by Cuillandre et al. at CFHT (http://www . cfht . hawaii . edu/jcc/Flips/flips.html). It is composed of Clanguage programs and $\mathrm{C}$-shell commands that automatically generate master bias, dark and flat images, build a model of the fringe pattern and subtract the master bias, dark files and fringe pattern as well as the overscan from raw $\mathrm{CFH} 12 \mathrm{~K}$ images. Each pixel and each image is then rescaled to account for intrinsic efficiency. A binary mask image provided by CFHT at each observing run identifies all bad and hot pixels over each CCD of the camera. They are taken into account during the master file generation process. The final data products are pre-calibrated images which include the description of the preprocessing history in the FITS header and the value of the CCD to $\mathrm{CCD}$ rescaling coefficient, according to the different gains of each CCD output and the quantum efficiency difference 
Table 2. Total area covered for each of the four survey fields.

\begin{tabular}{|c|c|c|c|}
\hline Field & Filter & $\begin{array}{c}\text { Total Area } \\
\left(\mathrm{deg}^{2}\right)\end{array}$ & $\begin{array}{c}\text { Exp. time } \\
\text { (mn) }\end{array}$ \\
\hline 0230-04 & $B$ & 3.9 & 120 \\
\hline \multirow[t]{3}{*}{ "Wide" } & $V$ & 4.17 & 90 \\
\hline & $R$ & 3.6 & 60 \\
\hline & $I$ & 5.4 & 60 \\
\hline 0230-04 & $B$ & 1.45 & 420 \\
\hline \multirow[t]{3}{*}{ "Deep" } & V & 1.45 & 270 \\
\hline & $R$ & 1.45 & 180 \\
\hline & $I$ & 1.45 & 120 \\
\hline \multirow[t]{4}{*}{$1003+01$} & $B$ & 3.0 & 120 \\
\hline & V & 2.94 & 90 \\
\hline & $R$ & 2.94 & 60 \\
\hline & $I$ & 4.14 & 60 \\
\hline \multirow[t]{4}{*}{$1400+05$} & $B$ & 2.85 & 120 \\
\hline & $V$ & 2.85 & 90 \\
\hline & $R$ & 3.6 & 60 \\
\hline & $I$ & 4.32 & 60 \\
\hline \multirow[t]{4}{*}{$2217+00$} & $B$ & 1.14 & 120 \\
\hline & $V$ & 1.14 & 90 \\
\hline & $R$ & 1.14 & 60 \\
\hline & $I$ & 3.6 & 60 \\
\hline
\end{tabular}

between epitaxial and high resisitivty CCDs. At this stage, all CCDs are therefore in the same ADU scaling units and arbitrarily normalised to the CCD\#04 efficiency.

The FLIPS package has been installed at the Terapix center described below in order to handle the pre-processing locally.

\subsection{The Terapix facility}

The Terapix facility (http://terapix.iap.fr) has been set up as a French national center to handle the processing of large imaging cameras. The facility includes the hardware and software environment required to process data on a Terabyte scale. Three COMPAQ XP1000 workstations with up to 2 GB of RAM memory each connected to four Terabytes of hard disks installed in a secured raid-5 configuration have been devoted to the VIRMOS image processing (Mellier et al. 2002). Over the past four years, Terapix has processed more than $10000 \mathrm{CFH} 12 \mathrm{~K}$ images for the VIRMOS survey, totalling about $2.5 \mathrm{~TB}$ of input data. A database environment allows for the streamlining of data access and storage.
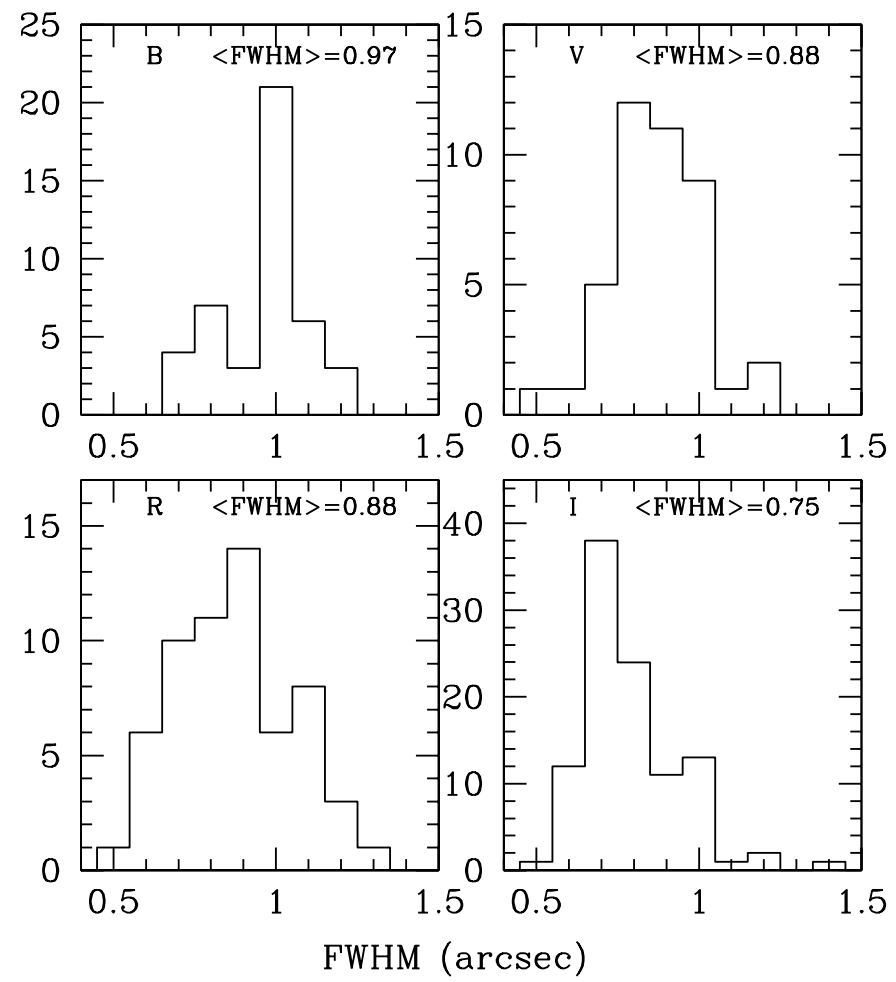

Fig. 3. Image quality $F W H M$ measured on point sources for all survey images acquired in $B$ (top left), $V$ (top right), $R$ (bottom left), I (bottom right).

\subsection{Pipeline processing}

Pipeline processing allows efficient data processing with excellent quality control. The stable behavior of the CFH12k camera allows for very accurate bias, flat field, and fringing correction.

The pipeline software has been developed within the Terapix environment. The main steps are described in McCracken et al. (2003) as well as in the Terapix progress reports (Mellier et al. 2002). The software tools developed for the object detection, image astrometry, photometry, pixel weighting and flagging, image resampling and stacking, object classification and catalogue construction are described at http://terapix.iap.fr/soft/ and can be downloaded freely from this site. The http://terapix.iap.fr/soft/releases.html also provide a documentation for all software tools.

The astrometric projection of all images into a common system used to build the large mosaic images has been developed to ensure an overall absolute calibration to the USNO reference catalogue (Monet et al. 1998) accurate to better than 0.3 arcsec, while the relative position accuracy within the catalogs is better than 0.1 arcsec rms The photometric calibration ensures that all individual images are calibrated on the same reference. Photometric zero point uncertainties are better than 0.1 magnitudes across the final mosaics. A detailed description and application of the pipeline, with a comprehensive description of quality assessments are presented in (McCracken et al. 2003). Similar procedures applied to the wide VIRMOS survey and the deep $U$-band survey have been used by Gwyn et al. (2003, in preparation) and Radovich et al. (2003), respectively. 


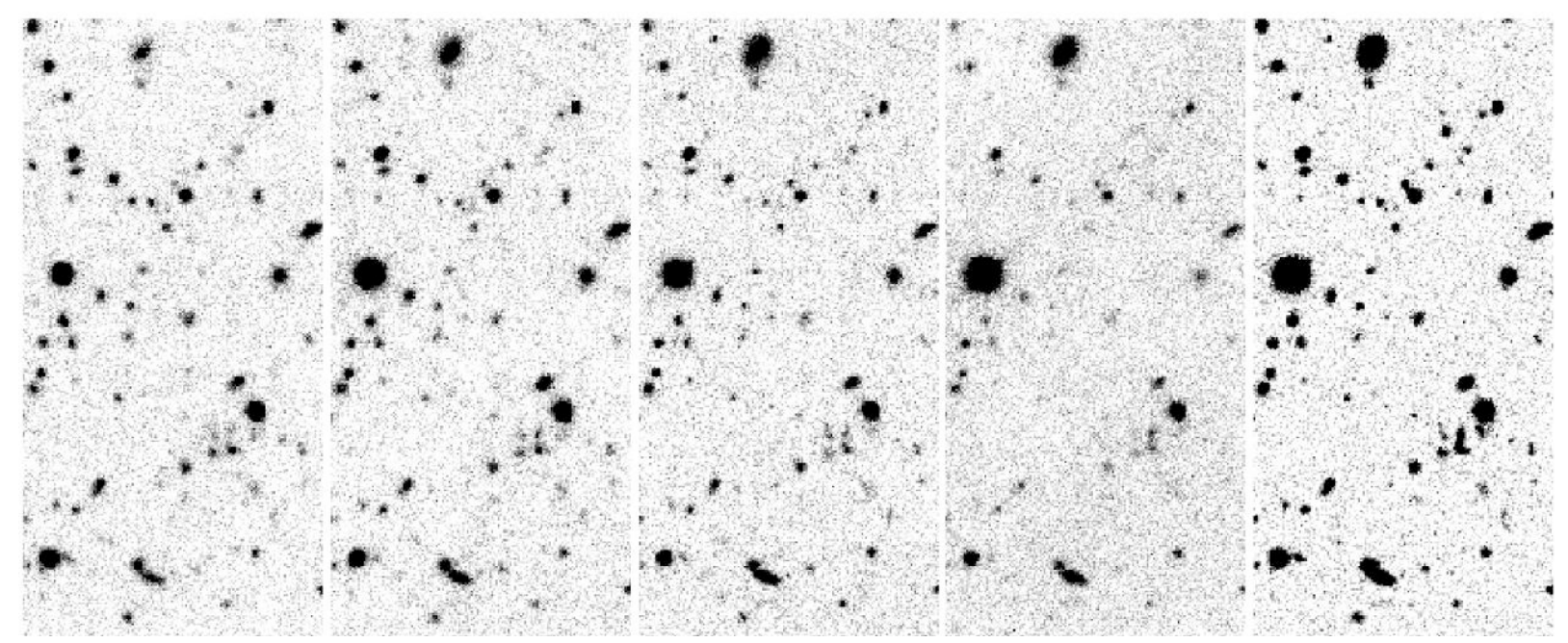

Fig. 4. Illustration of the final BVRI and $\chi^{2}$-images generated for the VIRMOS "deep" survey. The $\chi^{2}$-image is deeper and show sharper detections than the individual filter images. Each image is $\sim 3 \times 4 \operatorname{arcmin}^{2}$.

\section{Results: Survey images and catalogs}

\subsection{Catalog content}

The catalogs are built using the SEXTRACTOR software (Bertin $\&$ Arnouts 1996). Particular care has been taken to ensure uniform source detection, using a matched $\chi^{2}$ image (see Fig. 4) produced from the individual band images (McCracken et al. 2003; Szalay et al. 1999) for the "deep" field VVDS-0226-04 images, or a local threshold algorithm for the other "wide" fields. The difference between these two methods has been demonstrated to be marginal, with no effect in the magnitude range to the completness limit (McCracken et al. 2003).

Because of the large area covered, bright stars are present in the mosaics, creating increased local background. In addition, there are areas in the images not useable for accurate photometry. These can be areas for which the signal to noise is too low like e.g. areas of overlap between individual CCDs, or regions affected by internal reflecions or ghosts from bright stars around the field, or even areas affected by transient effects like satellite trails remaining at low $\mathrm{S} / \mathrm{N}$ after the median filtering. Masks are therefore applied to each mosaic. The area masked is is around $2-3 \%$ of the total area.

The catalogs contain the following parameters for each object generated from SEXTRACTOR V.2.3 used with the configuration files given in the Appendix and which were used as templates for all optical data from the CFH12K part of the survey:

- Identifier.

- Positions:

- X-IMAGE (pixel), Y-IMAGE (pixel);

- ALPHA_J2000 (decimal degrees) and DELTA_J2000 (decimal degrees).

- Flux and Magnitudes in the AB photometric system, defined as $-2.5 \log$ (flux in ADU)+ZP (mag zero point), including the associated errors:

- the total flux and magnitude FLUX_AUTO (ADU), MAG_AUTO;
- the isophotal flux and magnitude, FLUX_ISO (ADU), MAG_ISO;

- the corrected isophotal flux and magnitude FLUX_ISOCOR (ADU), MAG_ISOCOR;

- the best of MAG_AUTO and MAG_ISOCOR, FLUX_BEST (ADU), MAG_BEST;

- the aperture flux and magnitude FLUX_APER(2) (ADU), MAG_APER(2).

We compute colours using total magnitudes, in order to provide an accurate estimate of galaxy colour for bright, large objects.

- Shapes:

- image size, KRON_RADIUS (pixel) and ISOAREA_IMAGE $\left(\right.$ pixel $\left.^{2}\right)$;

- major axis A_IMAGE (pixel), A_WORLD (decimal degrees);

- minor axis B_IMAGE (pixel), B_WORLD (decimal degrees);

- position angle of main axes (all angles are measured counter-clockwise from the east. THETA denotes the position angle between the major axis, "A", and the NAXIS1 (east-west) image axis), THETA_IMAGE (decimal degrees), THETA_J2000 (decimal degrees);

- the values of the weighted second moment matrix coefficients, X2_IMAGE (pixel $\left.{ }^{2}\right), \quad$ Y2_IMAGE $\left(\mathrm{pixel}^{2}\right)$, XY_IMAGE $\left(\right.$ pixel $\left.^{2}\right)$;

- X2_WORLD (decimal degees ${ }^{2}$ ), Y2_WORLD (decimal degees $^{2}$ ), XY_WORLD (decimal degees ${ }^{2}$ );

- peak surface brightness MU_MAX (mag/arcsec $\left.{ }^{2}\right)$;

- detection threshold above the background MU_THRESHOLD (mag/arcsec ${ }^{2}$ ).

- Flags: several flags (FLAGS) are stored for each object. they indicate:

- saturated pixels;

- pixel located inside a masked area, as defined above;

- star-galaxy classification: a star/galaxy classifier defined from the half-light radius vs. magnitude diagram 
(see McCracken et al. 2003). Stars are selected along the vertical branch at fixed radius corresponding to seeing disk size. This classical method (described e.g., in Butchins 1982) turns out to be more reliable than the CLASS_STAR provided by SEXTRACTOR.

The photometric and astrometric quality have been assessed through many quality checks. The positional accuracy has been checked against the USNO catalog. The photometric accuracy has assessed through the Elixir queue observing program part of the queue observations at CFHT, which monitors the photometric zero points over many nights. The magnitudes of objects common to adjacent CCDs have been compared and have differences in the range $0.05-0.1 \mathrm{mag}$. The comparison of colors for the bright stellar objects in the fields with the observed and predicted locus of stars has shown color errors less than 0.1 mag. Counts of galaxies are in full agreement with the literature. Finally, the angular correlation function shows similar shape and amplitude as from other imaging surveys. All these quality checks are extensively described in McCracken et al. (2003). They ensure that the data are free of systematics before science analysis can be conducted.

In the $I$ band, a total of 2.175 million objects have been detected. Among these, 1.15 million objects have $17.5 \leq I_{\mathrm{AB}} \leq$ 24 , in the range of the VIRMOS-VLT Deep Survey.

An example of a multi-color set is given in Fig. 4.

\subsection{Database access}

All catalogs and mosaic images are stored in an interactive database implemented under the Oracle- 8 environment. Specific database development have been conducted to allow for easy database query and data retrieval.

The catalogs are now directly accessible to the CFHT-VIRMOS consortium and to general CFHT users (http://www.oamp.fr/cencos). To access the database, first a password should be requested through the online registration web page. This database allows users to query the photometric catalog and to retrieve image sections. Any parameter listed in the photometric catalog can be used to define a user selection, e.g. magnitudes, colors, and/or positions. A user catalog is then produced with entries as selected by the user from the list of catalog parameters.

\section{Conclusions}

A deep imaging survey has been conducted in four high galactic latitude areas with the $\mathrm{CFH} 12 \mathrm{k}$ camera. A total area of more than $17 \mathrm{deg}^{2}$ has been imaged in $I$ band, and significant coverage of the survey area has been performed also in $B, V$ and $R$. Pipeline processing has been conducted at the Terapix facility at IAP to produce large mosaic images calibrated in astrometry and photometry.

Photometric catalogs containing positions, magnitudes, colors, shapes of more than 2 million objects have been produced for more than $17 \mathrm{deg}^{2}$ and quality control has been applied as described in joint papers. These catalogs and mosaic images will be released for general use starting July 1st, 2003, at http://www . oamp. fr/virmos.

While many scientific programs are under way, the survey has already been used for cosmic shear studies. The VIRMOSDESCART CFHT survey (http://www.terapix.iap.fr/ Descart/cfhtsurvey.html) primarily focusses on the I-band sample to measure galaxy shape parameters with high accuracy and to probe the dark matter properties and cosmological parameters. Because these cosmic shear results provide also an estimate of systematics on galaxy shapes and can be cross-checked with independent cosmic shear results from other teams, they also provide quality assessments on the VIRMOS data set. In addition to the detection of cosmic shear signal (van Waerbeke et al. 2000), constraints on $\Omega_{m}, \sigma_{8}$ (van Waerbeke et al. 2002), the biasing properties of the dark matter (Hoekstra et al. 2002; Pen et al. 2003a) as well as its non-Gaussian properties (Bernardeau et al. 2002; Pen et al. 2003b) and the 3-dimension power spectrum of the dark matter (Pen et al. 2003a) have already been provided using the VIRMOS survey for cosmic shear.

The VIMOS spectroscopic survey has now started at the ESO/VLT and more than 20000 spectra have been collected (Le Fèvre et al. 2003). The joint $U$-band, optical, near infrared and spectroscopic information is now under process to probe the star formation history, the clustering history of galaxies up to redshift $z \approx 5$ and the properties of the light and dark matter relations.

Acknowledgements. We thank the CFHT time allocation committee for continuous support of this long term program, the CFHT staff for the execution of the observations performed in queue scheduling mode, the Terapix staff for its continuous help during the VIRMOS image processing period, the CNRS-INSU, CEA/DAPNIA and the Programme National de Cosmologie for support of the Terapix facility and IAP and OAMP for funding of this program.

\section{References}

Bernardeau, F., Mellier, Y., \& van Waerbeke, L. 2002, A\&A, 389, 28 Bertin, E., \& Arnouts, S. 1996, A\&AS, 117, 393

Bondi, M., Ciliegi, P., Zamorani, G., et al. 2003

[arVix: astro-ph/03030364]

Boulade, O., Charlot, X., Abbon, P., et al. 2000, Optical and IR telescope instrumentation and detectors, ed. M. Iye, \& A. Moorwood, Proc. SPIE, 4008, 657

Butchins, S. A. 1982, A\&A, 109, 360

Cuillandre, J.-C., Luppino, G. A., Starr, B. M., \& Isani, S. 2000, in Optical and IR telescope instrumentation and detectors, ed. M. Iye, \& A. Moorwood, Proc. SPIE, 4008, 1010

Groom, D. E. 2000, in Optical and IR telescope instrumentation and detectors, ed. M. Iye, \& A. Moorwood, Proc. SPIE, 4008, 634

Hoekstra, H., van Waerbeke, L., Gladders, D., \& Mellier, Y. 2002, ApJ, 577, 604

Iovino, A., Garilli, B., Scodeggio, M., et al., in preparation

Kuijken, K., Bender, R., Cappellaro, E., et al. 2002. The Messenger, 110,15

Landolt, A. U. 1992, AJ, 104, 340

Le Fèvre, O., Vettolani, G., Maccagni, D., et al. 2003, The Messenger, 111,18 
McMahon, R. G., Walton, N. A., Irwin, M. J., et al. 2001, New Astron. Rev., 45, 97

McCracken, H. J., Le Fèvre, O., Brodwin, M., et al. 2001, A\&A, 376, 756

McCracken, H. J., Radovich, M., Bertin, E., et al. 2003, A\&A, 410, 17

Mellier, Y., Bertin, E., Bonnarel, F., et al. 2002, Terapix Progress Report, http://terapix.iap.fr/doc/doc.html

Monet, D., et al. 1998, A Catalog of Astrometric Standards, US Naval Observatory

Miyazaki, S., Komiyama, Y., Sekigushi, M., et al. 2002, PASJ, 54, 833

Nonino, M., Bertin, E., da Costa, L., et al. 1999, A\&AS, 137, 51

Pen, U. L., Zhang, T., van Waerbeke, et al. 2003b, ApJ, ApJ, 592, 664
Pen, U. L., Lu, T., van Waerbeke, L., \& Mellier, Y. 2003a, MNRAS, 346, 994

Pierre, M., Valtchanov, I., Dos Santos, S., et al. 2003 [arVix:astro-ph/0305191]

Postman, M., Lauer, T. R., Szapudi, I., \& Oegerle, W. 1998, ApJ, 506, 33

Radovich, M., Arnaboldi, M., Ripepi, V., et al., in preparation Schlegel, D. H., Finkbeiner, D. P., \& Davis, M. 1998, ApJ, 500, 525 Szalay, A. S., Connolly, A. J., \& Szokoly, G. P. 1999, AJ, 117, 68 van Waerbeke, L., Mellier, Y., Erben, T., et al. 2000, A\&A, 358, 30 van Waerbeke, L., Mellier, Y., Pello, R., et al. 2002, A\&A, 393, 369 van Waerbeke, L., \& Mellier, Y. 2003 [arVix: astro-ph/0305089] Wilson, G. 2003, ApJ, 585, 191 


\section{Online Material}




\section{O. Le Fèvre et al.: VIRMOS imaging survey. I., Online Material p 2}

\section{Appendix A: SEXTRACTOR configuration files}

Tables A.1 and A. 2 list the parameters used for all $\chi^{2}$-images and all wide field analysis of VIRMOS optical obervations with the $\mathrm{CFH} 12 \mathrm{~K}$. 
Table A.1. SEXTRACTOR configuration files used for the F02 deep survey, $\chi^{2}$-image.

\begin{tabular}{|c|c|c|}
\hline \multicolumn{3}{|c|}{$\begin{array}{l}\text { \#Default configuration file for SEXTRACTOR V1.2B14 } \\
\text { \# EB 23/07/98 } \\
\text { \# (*) indicates parameters which can be omitted from this config file. } \\
\text { \#-- }\end{array}$} \\
\hline CATALOG_NAME & 02hr_chisq.cat & \# name of the output catalog \\
\hline CATALOG_TYPE & ASCII_HEAD & $\begin{array}{l}\text { \# "NONE","ASCII_HEAD","ASCII",'FITS_1.0" } \\
\text { \# or "FITS_LDAC" }\end{array}$ \\
\hline \multirow[t]{2}{*}{ PARAMETERS_NAME } & photom_new.param & $\begin{array}{l}\text { \# name of the file } \\
\text { \# containing catalog contents }\end{array}$ \\
\hline & \multicolumn{2}{|c|}{ \#-- } \\
\hline DETECT_TYPE & $\mathrm{CCD}$ & \# "CCD" or "РНOTO" (*) \\
\hline DETECT_MINAREA & 3 & \# minimum number of pixels above threshold \\
\hline DETECT_THRESH & & $\#<$ sigmas $>$ or $<$ threshold $>,<\mathrm{ZP}>$ in mag. $\operatorname{arcsec}^{-2}$ \\
\hline ANALYSIS_THRESH & & $\#<$ sigmas $>$ or $<$ threshold $>,<\mathrm{ZP}>$ in mag. $\operatorname{arcsec}^{-2}$ \\
\hline THRESH_TYPE & ABSOLUTE & \\
\hline FILTER & $\mathrm{N}$ & \\
\hline FILTER_NAME & gauss_3.0_7x7.conv & \# name of the file containing the filter \\
\hline DEBLEND_NTHRESH & & \# Number of deblending sub-thresholds \\
\hline DEBLEND_MINCONT & 0.002 & \# Minimum contrast parameter for deblending \\
\hline CLEAN & $\mathrm{Y}$ & \\
\hline CLEAN_PARAM & 1.0 & \# Cleaning efficiency \\
\hline MASK_TYPE & CORRECT & $\begin{array}{l}\text { \# type of detection MASKing: can be one of } \\
\text { \# "NONE", "BLANK" or "CORRECT" }\end{array}$ \\
\hline \multicolumn{3}{|c|}{ \#--1--1- } \\
\hline PHOT_APERTURES & $15 ., 25$. & \# MAG_APER aperture diameter(s) in pixels \\
\hline PHOT_FLUXFRAC & $0.2,0.5,0.8$ & \# Fraction of FLUX_AUTO defining FLUX_RADIUS \\
\hline PHOT_AUTOPARAMS & $2.5,3.5$ & \# MAG_AUTO parameters: <Kron_fact $>,<$ min_radius $>$ \\
\hline SATUR_LEVEL & 40000. & \# level (in ADUs) at which arises saturation \\
\hline PHOT_AUTOAPERS & $15.0,15.0$ & \# MAG_AUTO minimum apertures: estimation,photometry \\
\hline MAG_ZEROPOINT & 31.90 & \# magnitude zero-point \\
\hline MAG_GAMMA & & \# gamma of emulsion (for photographic scans) \\
\hline GAIN & & \# detector gain in $\mathrm{e}-/ \mathrm{ADU}$. \\
\hline PIXEL_SCALE & 0.205 & \# size of pixel in arcsec ( $0=$ use FITS WCS info). \\
\hline \multicolumn{3}{|c|}{ \#-- Star/Galaxy Separation } \\
\hline SEEING_FWHM & & \# stellar FWHM in arcsec \\
\hline STARNNW_NAME & default.nnw & \# Neural-Network_Weight table filename \\
\hline \multicolumn{3}{|c|}{ \#---1-- } \\
\hline BACK_SIZE & & \# Background mesh: $<$ size $>$ or $<$ width $>,<$ height $>$ \\
\hline BACK_FILTERSIZE & & \# Background filter: $<$ size $>$ or $<$ width $>,<$ height $>$ \\
\hline BACKPHOTO_TYPE & LOCAL & \# can be "GLOBAL" or "LOCAL" (*) \\
\hline BACKPHOTO_THICK & & \# thickness of the background LOCAL annulus $(*)$ \\
\hline BACK_TYPE & AUTO & \\
\hline BACK_VALUE & 0 & \\
\hline \multicolumn{3}{|l|}{ \#---------------- } \\
\hline CHECKIMAGE_TYPE & NONE & $\begin{array}{l}\text { \# can be one of "NONE", "IDENTICAL", } \\
\text { \# "BACKGROUND", "-BACKGROUND",'BACKGROUND_RMS", }\end{array}$ \\
\hline CHECKIMAGE_NAME & check.fits & \# Filename for the check-image $(*)$ \\
\hline \multicolumn{3}{|c|}{ \#-- Memory (change with caution!) } \\
\hline MEMORY_OBJSTACK & 2000 & \# number of objects in stack \\
\hline MEMORY_PIXSTACK & 5000000 & \# number of pixels in stack \\
\hline MEMORY_BUFSIZE & & \# number of lines in buffer \\
\hline \multicolumn{3}{|c|}{ 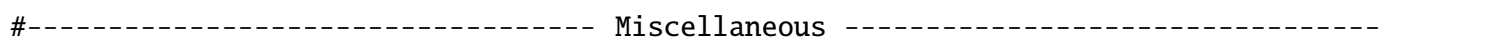 } \\
\hline VERBOSE_TYPE & NORMAL & \# can be "QUIET", "NORMAL” or "FULL" (*) \\
\hline \multicolumn{3}{|c|}{ \#---1--1, } \\
\hline WEIGHT_TYPE & MAP_WEIGHT & \\
\hline \#WEIGHT_TYPE & BACKGROUND & \\
\hline
\end{tabular}


Table A.2. SEXTRACTOR configuration files used for the wide field survey, using a flux detection threshold on the $I$-band image.

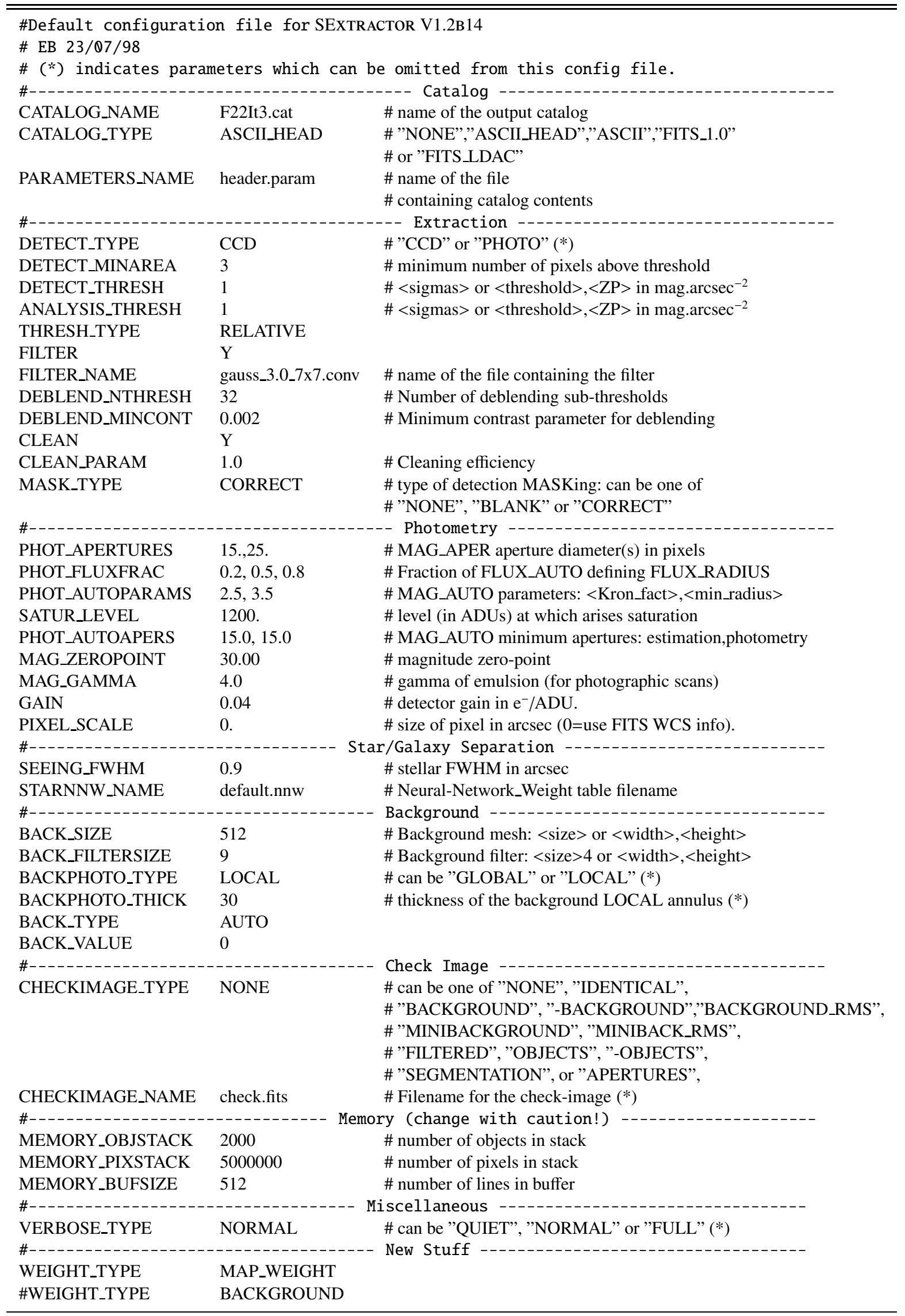

